EUROPEAN ORGANIZATION FOR NUCLEAR RESEARCH

CERN-PPE/97-96

10 July 1997

\title{
A PROGRESS REPORT ON THE DEVELOPMENT OF THE CSI-RICH DETECTOR FOR THE ALICE EXPERIMENT AT LHC
}

\author{
A. Braem, M. Davenport, A. Di Mauro, B. Goret, G. Paic, F. Piuz, \\ J. Raynaud, J.C. Santiard, S. Stucchi, T.D. Williams. \\ CERN, Geneva, Switzerland \\ N. Colonna, D. Di Bari, D. Elia, R. Fini, L. Galantucci, B. Ghidini, \\ A. Grimaldi, E. Monno, E. Nappi, F. Posa, G. Tomasicchio. \\ INFN and Dipartimento Interateneo di Fisica dell'Universitá di Bari, Italy
}

The ALICE HMPID group

\begin{abstract}
The particle identification in ALICE (A Large Ion Collider Experiment) at LHC will be achieved by two complementary systems based on time of flight measurement, at low $p_{t}$, and on the Ring Imaging Cherenkov (RICH) technique, at $p_{t}$ ranging from 2 to $5 \mathrm{GeV} / c$, respectively. The High Momentum PID (HMPID) system will cover $\sim 5 \%$ of the phase space, the single arm detector array beeing composed by seven $1.3 \times 1.3 \mathrm{~m}^{2} \mathrm{CsI}-\mathrm{RICH}$ modules placed at $4.7 \mathrm{~m}$ from the interaction point where a density of about 50 particles $/ \mathrm{m}^{2}$ is expected.

A $1 \mathrm{~m}^{2}$ prototype, 2/3 of HMPID module size, has been successfully tested at the CERN/PS beam where 18 photoelectrons per event have been obtained with $3 \mathrm{GeV} / \mathrm{c}$ pions and $10 \mathrm{~mm}$ liquid $\mathrm{C}_{6} \mathrm{~F}_{14}$ radiator. Mechanical problems related to the liquid radiator vessel construction have been solved and the prototype, fully equipped, will be tested at the CERN/SPS to investigate the PID capability in high particle density events.

In this report, after an introductory discussion on the requirements for PID in ALICE, the HMPID prototype is described and the main results of beam tests on large area CsI photocathodes, operated in RICH detectors, are given.
\end{abstract}

Presented by A. Di Mauro at the 7th Pisa Meeting on Advanced Detectors, May 25-31, 1997 - La Biodola, Isola d'Elba, Italy

Submitted to Nuclear Instruments and Methods in Physics Research A 
ALICE (A Large Ion Collider Experiment) is the only LHC experiment fully dedicated to the study of heavy ion collisions and it will address most of the hadronic and leptonic signals of QGP (Quark Gluon Plasma) formation. In ${ }^{207} \mathrm{~Pb}-{ }^{207} \mathrm{~Pb}$ collisions, at $2.76 \mathrm{TeV} /$ nucleon, low interaction rates $(\leq 10 \mathrm{KHz}$ with about $10 \%$ of central events $)$ and high multiplicities $(\sim 8000$ particles per rapidity unit) are expected. A detailed description of ALICE, whose detector is shown in fig. 1, can be found in [1,2].

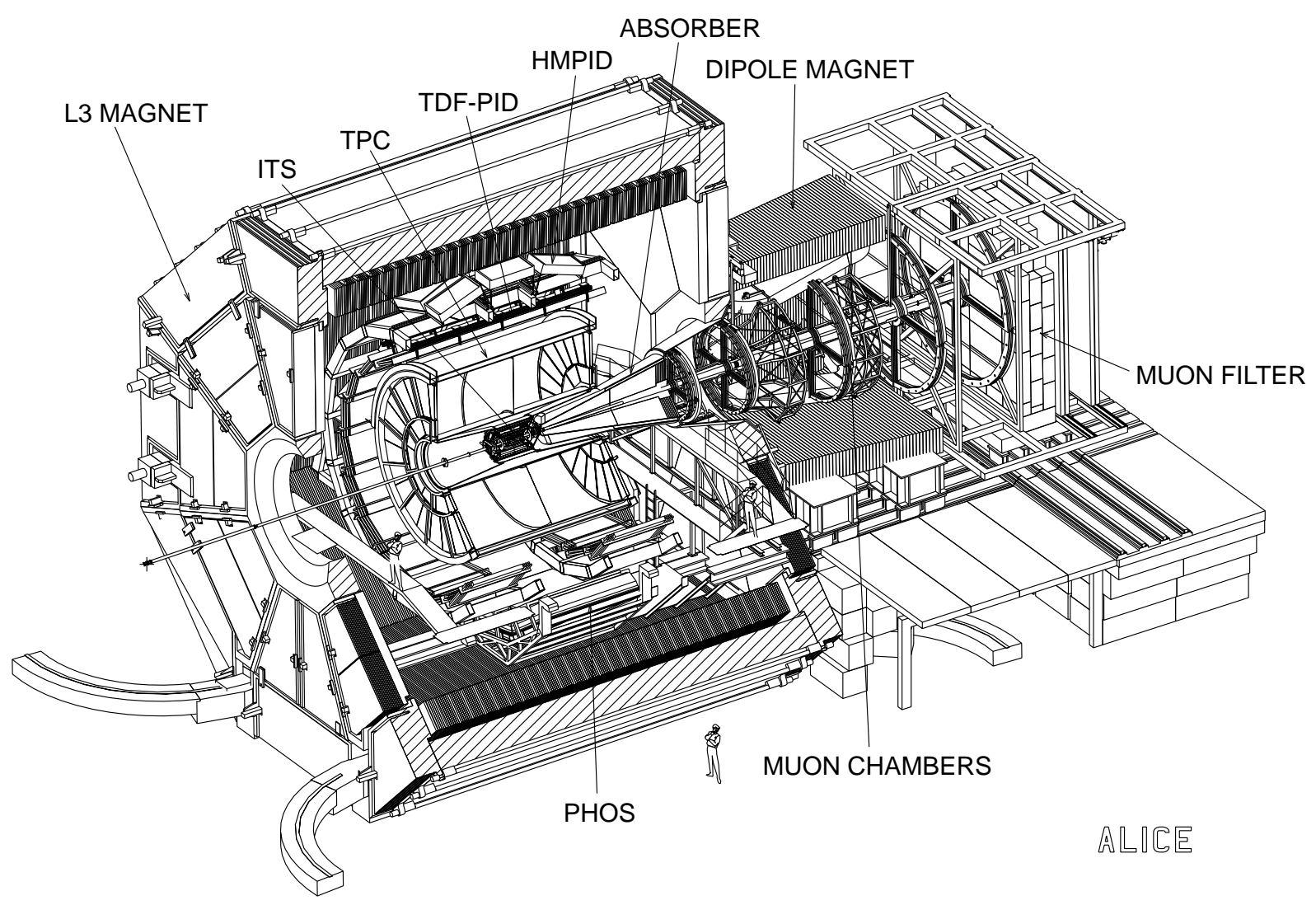

Figure 1: The ALICE detector layout. It consists of two main parts: a central device, embedded in the L3 magnet, with the Inner Tracking System (ITS), the TPC, the TOF barrel and the single arm HMPID and Photon Spectrometer systems, and a forward part with the muon spectrometer and two zero degree calorimeters (not shown in the figure) enabling the trigger on central collisions.

Particle identification (PID) is an important design feature in ALICE, because of the large spectra of momenta and masses that will be measured. In the momentum range below $2 \mathrm{GeV} / c$, where the bulk of hadrons is concentrated, the PID will be accomplished by TOF measurement in a barrel system. Only $3 \%$ of the produced hadrons will have $p_{t}$ larger than $2 \mathrm{GeV} / c$, a feature which makes this region accessible only to inclusive measurements. A dedicated High Momentum PID (HMPID) system has been designed to measure inclusive particle ratios and $p_{t}$ spectra 
in the range $2 \div 5 \mathrm{GeV} / c$. It is a single arm array of seven $1.3 \times 1.3 \mathrm{~m}^{2} \mathrm{CsI}-\mathrm{RICH}$ modules covering about $12 \mathrm{~m}^{2}$, namely $5 \%$ of full central acceptance (fig. 2). The detector segmentation and the tilting of the modules decrease the scatter of the incident angles of the particles (limiting them to a $0^{\circ} \div 15^{\circ}$ spread); this minimizes Cherenkov photons total internal reflection losses in the radiator and improves the identification capability. The RICH modules are placed at $4.7 \mathrm{~m}$ from the interaction vertex, position corresponding to multiplicities $\leq 50$ particles $/ \mathrm{m}^{2}$ (including expected background).

To verify the PID capabilities in such conditions of particle density a $1 \mathrm{~m}^{2}$ prototype has been built to be tested at the CERN/SPS. In this report a description of the prototype and main test beam results on large area CsI-RICH detector are presented.

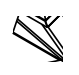

Figure 2: Perspective view of the HMPID system with the 7 RICH modules placed on top of the barrel frame and tilted according to the distance from the interaction point.

\section{The HMPID RICH prototype}

The RICH detector has a proximity focusing geometry, with $10 \mathrm{~mm}$ liquid $\mathrm{C}_{6} \mathrm{~F}_{14}$ radiator, suitable for the momentum range of interest, coupled through a $5 \mathrm{~mm}$ quartz window to a multiwire chamber (MWPC, $4 \mathrm{~mm}$ anode wire pitch, $2 \mathrm{~mm}$ anode-cathode gap) with a cathode segmented into $8 \times 8 \mathrm{~mm}^{2}$ pads for 2-D readout (fig. 3). A $500 \mathrm{~nm}$ photosensitive CsI film evaporated on the pads converts the Cherenkov photons into single electrons originating avalanches at the MWPC anode wires. The analog readout of the pads allows accurate localization through centroids evaluation. The use of a solid photoconverter permits thin sensitive gaps in the MWPC, resulting in a considerable reduction of the background from ionizing particles. This feature is mandatory with the expected multiplicity which makes the pattern recognition capability a prime requirement for our application. The photodetector can even be used as an additional tracking plane with good position resolution $[1,3]$.

The HMPID requirements make acceptance less crucial than performance, thus allowing some dead space in the detector plane. Therefore, to optimize the production procedure and to ease the handling, the photocathode (PC) and the radiator have been segmented into $64 \times 40 \mathrm{~cm}^{2}$ panels and $130 \times 42 \mathrm{~cm}^{2}$ vessels (fig. 4).

The HMPID prototype is $130 \times 85 \mathrm{~cm}^{2}$, namely $2 / 3$ of one RICH module size. It has been successfully tested at the CERN/PS test beam, demonstrating not only the good operation of the MWPC, having $1.3 \mathrm{~m}$ long anode wires and a support line in the middle, but also the feasibility and the mounting under clean controlled specifications of $64 \times 40 \mathrm{~cm}^{2}$ CsI PCs. The prototype will be completely equipped, with full size radiator vessels, to be tested at the CERN/SPS.

The designed radiator vessel consists of a NEO-CERAM (NIPPON GLASS, Japan) tray closed by a fused silica SUPRASIL window. The two materials have equal thermal dilation coefficients 


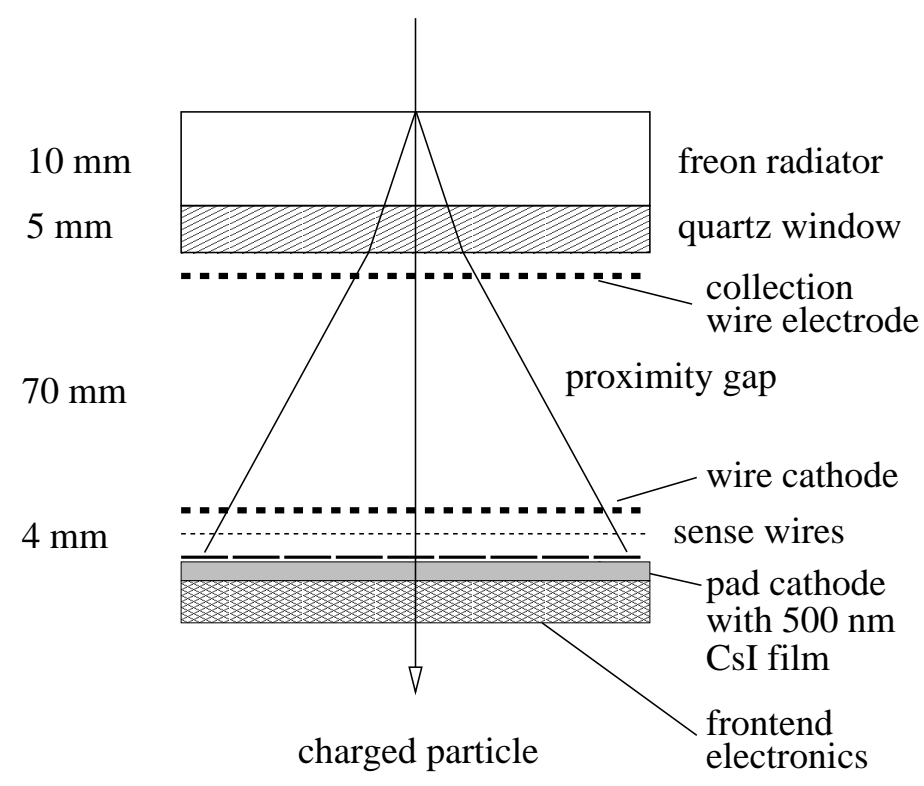

Figure 3: Schematic cross section of the HMPID CsI-RICH detector.

and the resulting container is characterized by the stiffness required to cope with the $\mathrm{C}_{6} \mathrm{~F}_{14}$ hydrostatic pressure. The vessel strain under hydrostatic load, in every position foreseen by the HMPID detector design, has been measured on a full size vessel and the results have confirmed the mechanical design expectations.

The ALICE low interaction rates allow multiplexed analog readout based on full custom electronics. Currently the frontend electronics of the RICH prototype is based on the 16 channel GASSIPLEX chip [4]. In the final detector version it will be based on the MultiChip Module (MCM) DIGITPLEX, composed by 4 GASSIPLEX's , the 10 bit ADC CRIAD and the zero suppression chip DILOGIC on a board [5]. The specifications are: ECN $<800 e^{-}$at $20 \mathrm{pF}$ input, baseline restoration $<0.5 \%$ after $3 \mu \mathrm{s}$, data ready after zero suppression for 64 channels in $3 \mu \mathrm{s}$.

\section{Test beam results on large area CsI-RICH detectors}

The HMPID requirements have directed the choice towards a fast-RICH configuration with CsI PC, which has been made possible by the CERN/RD26 project activities demonstrating the feasibility of large area CsI photocathodes with high and stable quantum efficiency [6]. The QE of 29 large PC's, produced at CERN, has been evaluated, exploiting the Cherenkov radiation in a RICH detector in test beam. A detailed presentation of the RD26 results can be found in [7].

Fig.5 shows the CsI QE measured at $170 \mathrm{~nm}$ as a function of the PC production number, summarizing the results of 4 years activity. The improvement can be attributed to the definition of a new procedure to produce the photocathodes. Firstly, the substrate is obtained by chemical deposition of nickel and gold layers on top of the copper pads, previously mechanically and chemically polished. Then the CsI is evaporated at $50{ }^{\circ} \mathrm{C}$ and $10^{-6}$ Torr, and the PC is kept 


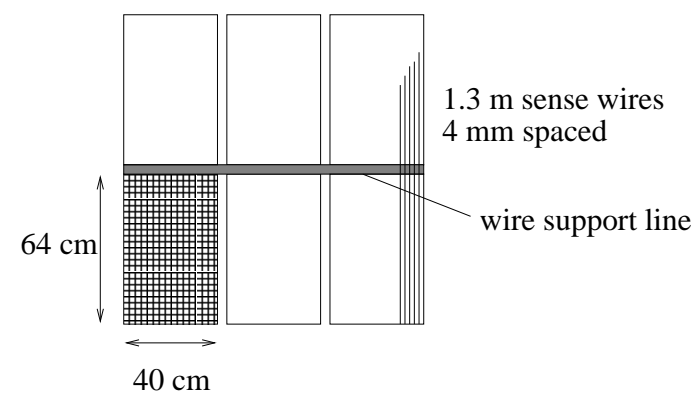

RADIATOR SEGMENTATION

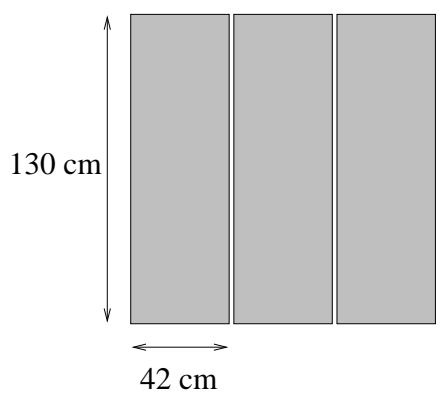

Figure 4: Segmentation of pad cathode and radiator.

under vacuum at $50{ }^{\circ} \mathrm{C}$ for six hours.

Another important feature is the QE stability: two PC's, among the best ones, kept under argon (between beam test periods) and periodically tested, show QE degradation less than 5\% in two years (fig.6).

Fig. 7 shows typical $3 \mathrm{GeV} / c$ pion events obtained at the CERN/PS test beam. Each PC is tested at several MWPC gains and Cherenkov radiator thickness and the photodetector performance is evaluated by means of dedicated analysis and simulation programs. The main event quantities are extracted by the analysis program from a fiducial region of the PC plane where all the Cherenkov photons hits are expected (figs. 8 and 9).

Then these quantities are reproduced by the Monte Carlo simulation, allowing to estimate CsI photoelectric yield and the photon feedback contribution. An essential feature, that the simulation program has permitted to observe, is the spatial distribution of feedback photons: due to the small anode-cathode gap and the pad size, they overlap to the cluster originated by the primary Cherenkov photon, thus resulting in a very small background.

The main test beam results can be summarized as follow:

1) a very stable, discharge-free, operation of the MWPC at a gain of $\sim 10^{5}$ and single electron detection efficiency of $95 \%$;

2) average number of 18 Cherenkov photoelectrons per ring with $3 \mathrm{GeV} / \mathrm{c}$ pion and $10 \mathrm{~mm}$ $\mathrm{C}_{6} \mathrm{~F}_{14}$ radiator;

3) single photon angular resolution of $8 \mathrm{mrad}$.

Furthermore two $80 \times 20 \mathrm{~cm}^{2}$ CsI photocathodes have been successfully used in the Threshold Imaging Cherenkov detector of the CERN/NA44 experiment, showing no degradation of the 


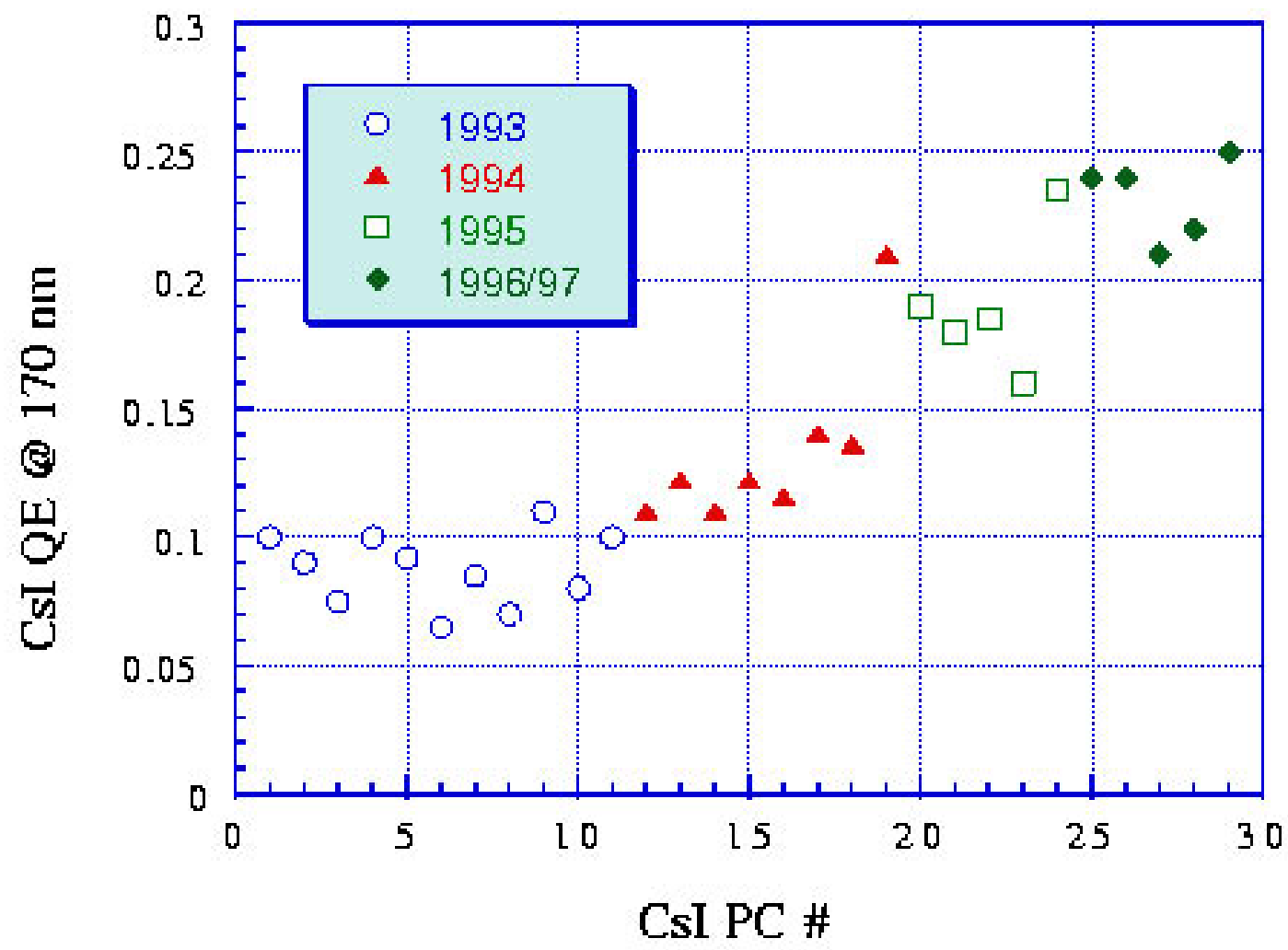

Figure 5: CsI QE measured at $170 \mathrm{~nm}$ as a function of the PC number. All the photocathodes have large area, from $30 \times 30 \mathrm{~cm}^{2}$ to $64 \times 40 \mathrm{~cm}^{2}$ of PC 28 and 29 which have been produced for the HMPID prototype. 


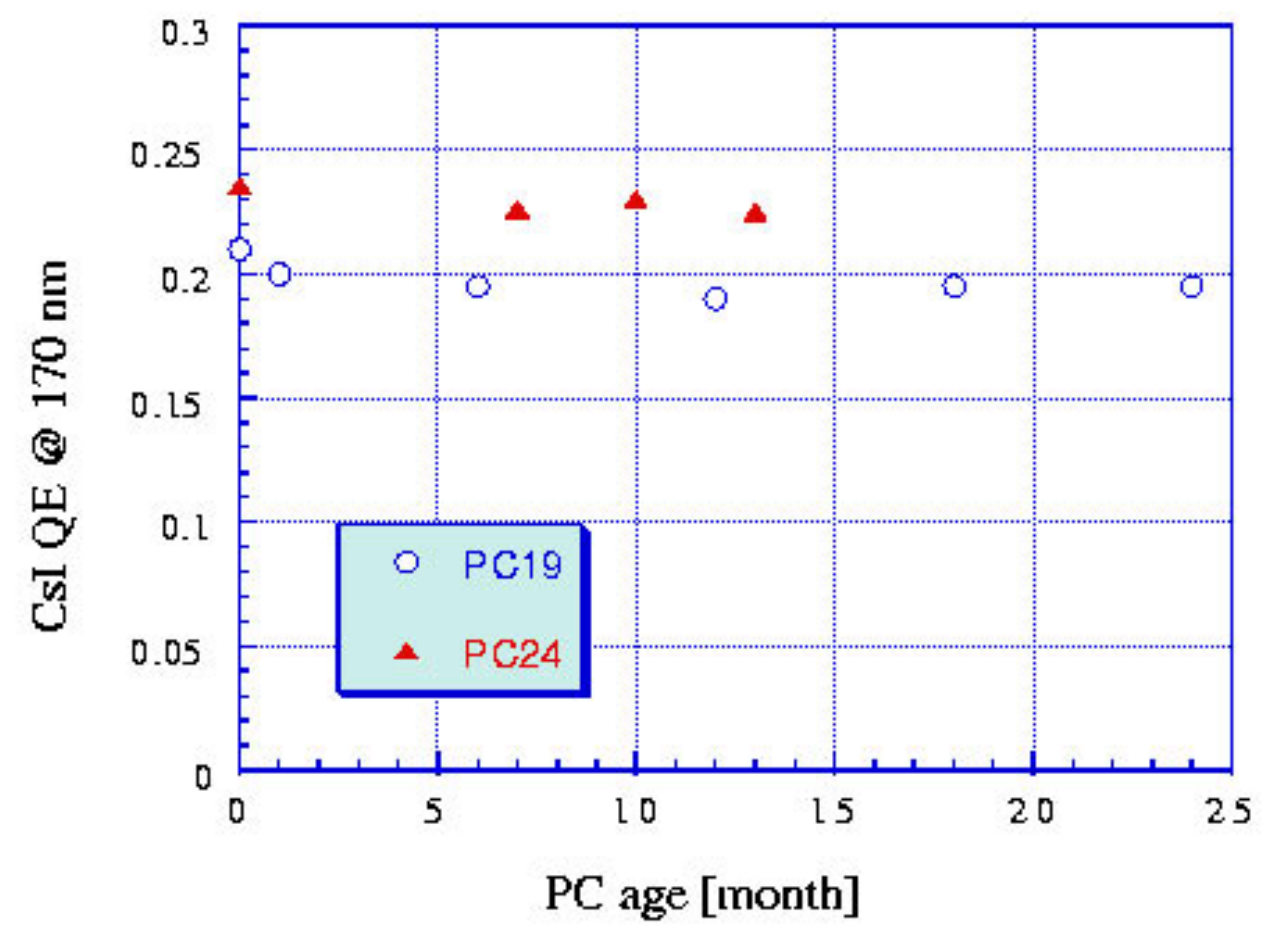

Figure 6: CsI QE measured at $170 \mathrm{~nm}$ as a function of PC age.

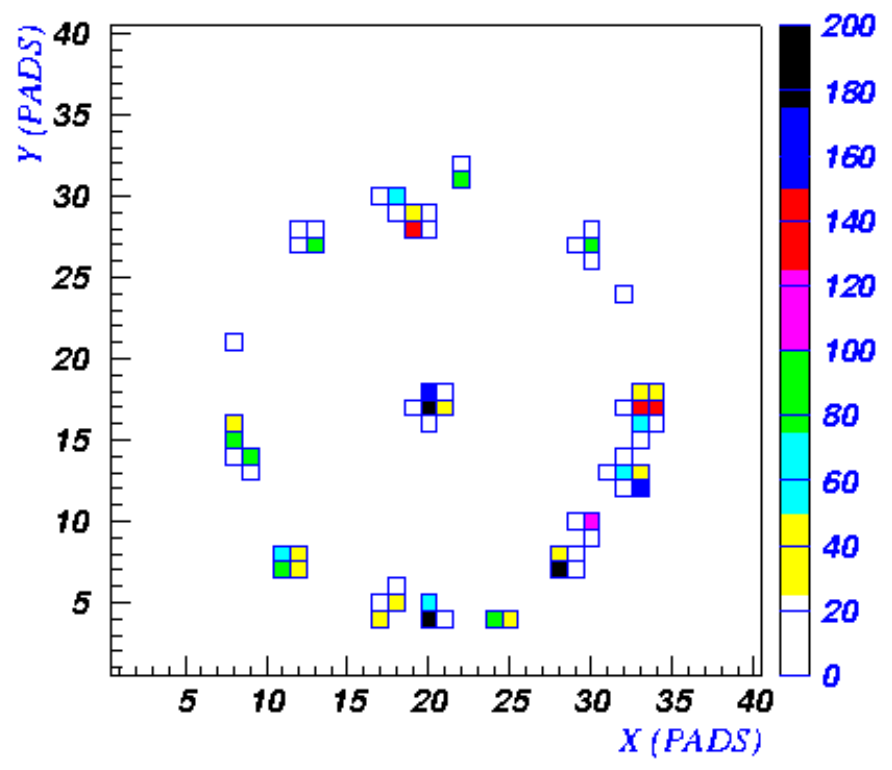

Figure 7: A $3 \mathrm{GeV} / c$ pion event, obtained with $\mathrm{PC} 24$ and $10 \mathrm{~mm}$ thick $\mathrm{C}_{6} \mathrm{~F}_{14}$ radiator, showing the pad cluster MIP surrounded by the Cherenkov photons ring. The ring radius of about $10 \mathrm{~cm}$ has been obtained with a proximity gap of $7 \mathrm{~cm}$. The grey scale on the right, in arbitrary units, is proportional to the signal measured on the pads. 


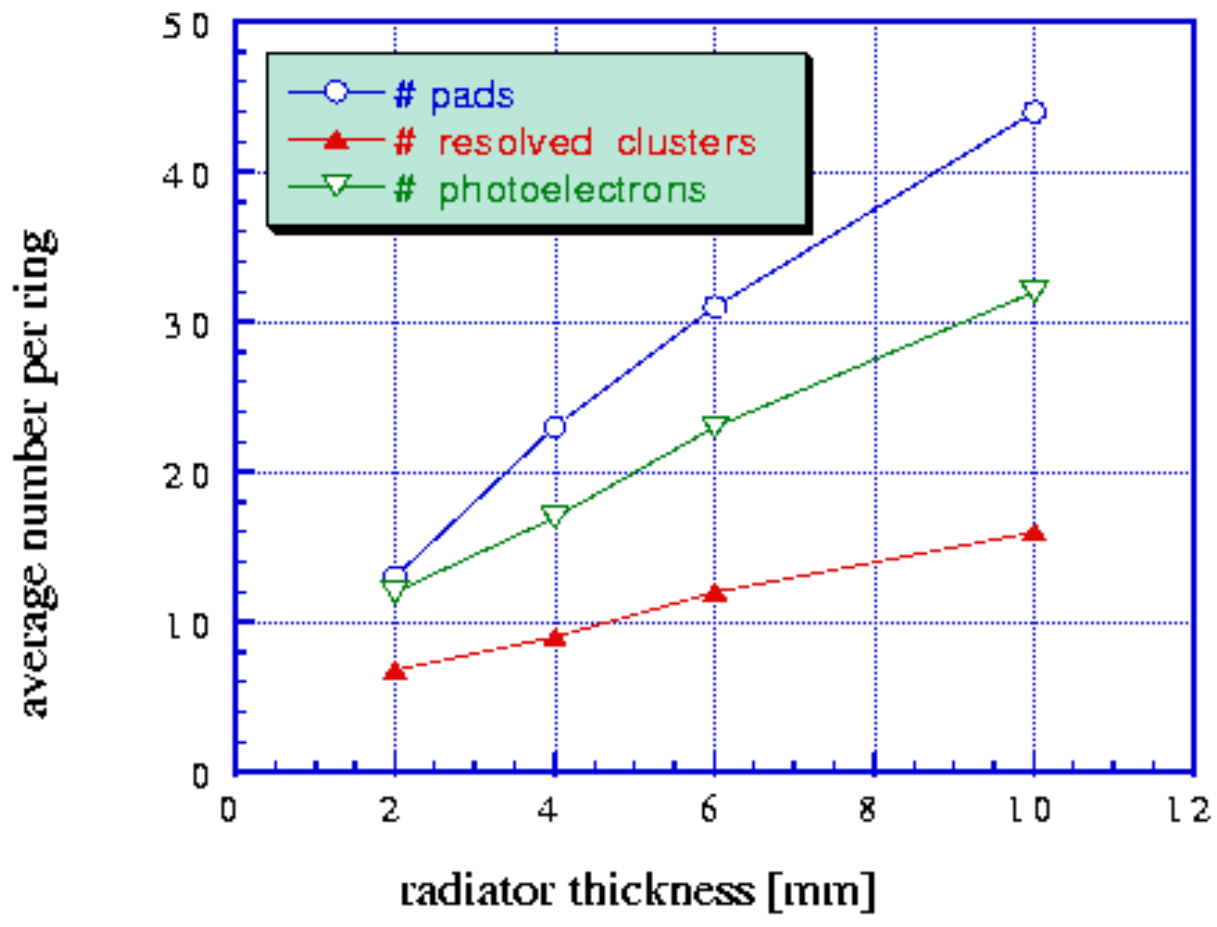

Figure 8: Average number of pads, pad clusters and photoelectrons as a function of the $\mathrm{C}_{6} \mathrm{~F}_{1} 4$ radiator thickness, at a gain of $10^{5}$ and with $3 \mathrm{GeV} / c$ pions. The number of photoelectrons, obtained as ratio between the total pulse height and the single electron pulse height, includes Cherenkov and feedback photons, while the number of resolved clusters is related to Cherenkov events. The mean number of pads per event depends on the cluster size, which varies roughly from 2 to 3 pads in the $2 \div 10 \mathrm{~mm}$ radiator thickness range. The cluster size increase with radiator thickness can be attributed to photoelectrons overlapping, which enhances the signal induced on pads and therefore the number of pads beeing over threshold. 


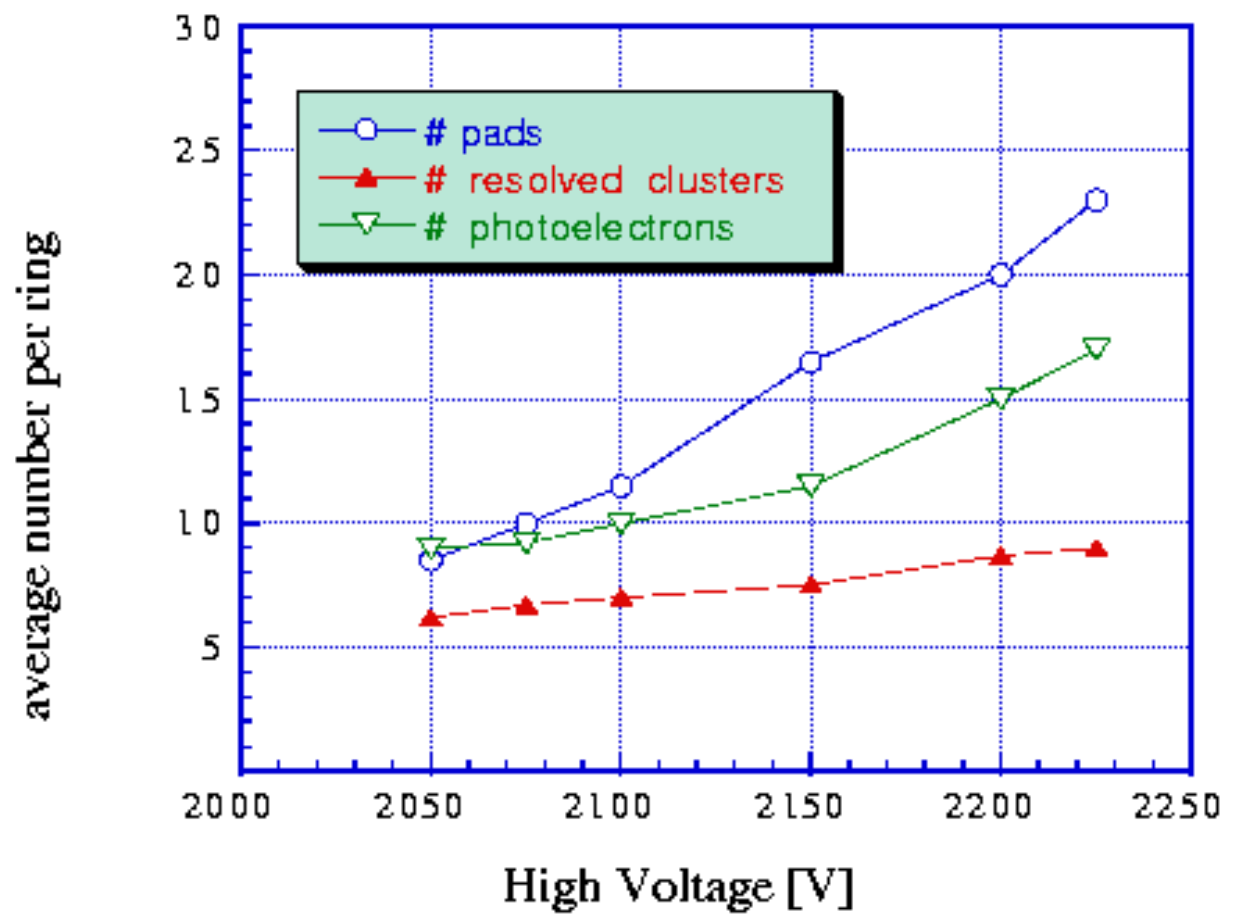

Figure 9: Average number of pads, pad clusters and photoelectrons as a function of the MWPC high voltage, at a radiator thickness of $4 \mathrm{~mm}$ and with $3 \mathrm{GeV} / c$ pions. The number of photoelectrons, obtained as ratio between the total pulse height and the single electron pulse height, includes Cherenkov and feedback photons, while the number of resolved clusters is related to Cherenkov events. Finally the mean number of pads per event depends on the cluster size which varies from from 1.5 pads, at $2050 \mathrm{~V}$ (i.e. at a gain of $1.5 \times 10^{3}$ ), to 3 pads, at $2225 \mathrm{~V}$ (i.e. at a gain of $7 \times 10^{4}$ ). The cluster size increase with chamber gain is due to the onset of photon feedback, as shown by the change of slope of the number of photoelectrons curve. 
performance during four weeks of high irradiation level in lead ion run [8].

\section{Conclusions}

The status of the development of the CsI-RICH detector for the HMPID in ALICE at LHC has reached a very important phase. A prototype, 2/3 of one final RICH module, has been built, confirming mechanical design expectations and showing also good performance of the MWPC with large area CsI photocathode. The foreseen test at the CERN/SPS will be of prime importance to assess the PID capability in events with high particle densities.

\section{References}

[1] ALICE Collaboration, Technical Proposal, CERN/LHCC 95-71

[2] ALICE Collaboration, Addendum to the Technical Proposal, CERN/LHCC 96-32

[3] E. Nappi et al., High Momentum PID with RICH detectors in the ALICE experiment at LHC (to be published in the proceedings of the International Conference on High Energy Physics ICHEP96 - Warsaw 1996, World Scientific, Singapore)

[4] J. C. Santiard, Gassiplex, a low noise analog signal processor for readout of gaseous detectors, CERN-ECP/94-17

[5] J. C. Santiard, MCM-Digitplex, a compact on-detector analog to digital processor, (published in the Proceedings of the 2nd Workshop on electronics for LHC experiments, Balaton 96)

[6] RD26 Collaboration, Status Report, CERN/LHCC 96-20

[7] F. Piuz, CsI-photocathode and RICH detector, Nuclear Instruments and Methods A 371 (1996) 96-115

[8] A. Braem et al., A Threshold Imaging Cherenkov Detector with CsI Photocathodes, to be published in the proceedings of the 7th Pisa Meeting on Advanced Detectors, May 25-31, 1997 - La Biodola, Isola d'Elba, Italy. 